also expressed by a number of cells in the inflammatory system. It is likely that release of osteopontin in joint destruction will influence the inflammatory response.

Other bone proteins with a capacity to bind $\alpha v \beta 3$ include BSP and osteoadherin. Their role in bone turnover and interactions with other tissues remains to be elucidated, but available data indicate primary functions in bone formation.

\section{SP0099 SYNOVIAL FIBROBLASTS DEGRADING CARTILAGE AND BONE}

${ }^{1,2} \mathrm{~T}$ Pap, ${ }^{1} Y$ Shigeyama, ${ }^{1,3} \mathrm{U}$ Müller-Ladner, ${ }^{1} \mathrm{~S}$ Ohtsu, ${ }^{4} \mathrm{~W}$ Van der Laan, ${ }^{5} \mathrm{~K}$ Aupperle, 1,3 J Schedel, ${ }^{6}$ W Aicher, ${ }^{5} \mathrm{G}$ Firestein, ${ }^{1} \mathrm{RE}$ Gay, ${ }^{1} \mathrm{~S}$ Gay. ${ }^{1}$ Center of Exp. Rheum., Department Rheum., University Hospital Zurich, Switzerland; ${ }^{2}$ Department of Exp. Rheum., University Hospital Magdeburg, Germany; ${ }^{3}$ Department of Medicine I, University Hospital Regensburg, Germany; ${ }^{4}$ Leiden University Medical Center, Leiden, The Netherlands; ${ }^{5}$ Division Rheumatology, Allergology and Immunology, UCSD School of Medicine, San Diego, USA; ${ }^{6}$ Bas Science Res. Lab., Department Orthop. Surg., University Hospital Tubingen, Germany

10.1136/annrheumdis-2001.43

The progressive destruction of cartilage and bone is one of the most characteristic features of rheumatoid arthritis (RA). It results form the concerted action of different cells types such as $\mathrm{T}$ cells, fibroblasts and macrophages that through direct contact as well as the release of cytokines form a cellular network in the inflamed synovium. Research of the last decade has provided evidence that RA synovial fibroblasts (RA-SF) contribute significantly to disease and play a major role in both initiating and driving the destructive process. It has been understood that intrinsic cellular activation, alterations in apoptosis and the attachment to cartilage and bone are critical prerequisites for the degradation of extracellular matrix.

The degradation of articular cartilage by activated RA-SF has been investigated in different in vitro and animal models. Among the latter, the SCID mouse model of RA has been used intensively to clarify the specific role of synovial fibroblasts in rheumatoid cartilage destruction. In this model, RA-SF are coimplanted with normal articular cartilage into severe combined immunodeficient (SCID) mice. As these mice do not reject the implants, the model allows studying the degradation of articular cartilage by RA-SF in the absence of human inflammatory cells. From the SCID mouse model it has been learnt that RA-SF maintain their activated phenotype in the absence of continuous inflammatory stimulation and progressively degrade cartilaginous matrix. As demonstrated by gene transfer experiments, modulation of intracellular signalling pathways such as the Ras-RafMAPK pathway, the NF-kappa B transcription factor and the p53 tumour suppressor gene alter the aggressive behaviour of RA-SF in the SCID mouse model of RA.

However, research has also demonstrated clearly that proinflammatory cytokines as produced by various cells in the rheumatoid synovium further enhance the aggressive potential of RASF. Apart form TNF alpha, IL-1, and IL-1 dependent pathways appear to play a central role.

It is now well established that RA-SF destroy the articular cartilage directly through the release of matrix degrading enzyme such as matrix metalloproteinases (MMPs), cathepsins and serine proteases. While several of these enzymes have been identified in the rheumatoid synovium and demonstrated to contribute to cartilage degradation, the specific role of individual members of these families is still poorly understood. In addition, despite a clear understanding of general mechanisms that regulate the expression of MMPs and cathepsins, specific pathways by which these enzymes are upregulated differentially in the course of disease need clarification. Current efforts focus on the modulation of enzymes that apart from degrading extracellular matrix directly, play a major role in activating other matrix degrading enzymes. Membrane-type MMPs (MT-MMPs) as well as the Plasmin system appear promising candidates for such approaches.

So far, fibroblasts have been associated with bone resorption mainly by indirect mechanisms such as the release of factors that enhance osteoclast activity and facilitate the differentiation of macrophages into osteoclast-like cells. Thus, fibroblasts of the rheumatoid synovium constitute a major source of the osteoclast differentiating factor (ODF) that appears to play a central role in the differentiation and activation of osteoclasts. However, recent data indicate that specific populations of activated fibroblasts such as from the synovial like interface membrane around loose joint prostheses are also capable of resorbing bone in vivo without the help of osteoclasts. Upon stimulation, such fibroblasts release large amounts of acidic components that may account for their ability to decalcify bone prior to its degradation. Using RTPCR, in situ hybridization and immuno- transmission electron microscopy, we have been able to demonstrate that these cells express a vacuolar type ATPase (v-ATPase) on their cell surface that has been implicated in the $\mathrm{H}^{+}$secretion by different cell types. It is concluded that subtypes of activated fibroblasts may not only enhance but also actively contribute to the bone resorption. The question of whether this applies also to RA-SF or is associated with different ways of cellular activation needs to be determined.

\section{Review session $\mathrm{N}^{\circ}$ 4: The kidney in systemic R. D. - Saturday 16 June, 10.00-11.45/ Forum Hall}

\section{SP0100 PATHOLOGIC CLASSIFICATION OF LUPUS NEPHRITIS}

D Ferluga. Institute of Pathology, Faculty of Medicine University of Ljubljana, Ljubljana, Slovenia

10.1136/annrheumdis-2001.44

A myriad of autoantibodies, particularly those against dsDNA, have been found to be involved in the production of a large heterogeneous set of immune complexes whose deposition in various organs, especially kidneys, appears to play a major role in triggering tissue lesions and an inflammatory response in systemic lupus erythematosus (SLE). In our series of 379 kidney biopsy and autopsy samples from SLE patients, immune deposits were demonstrated by traditional immunofluorescence in the glomeruli (99.0\%) and characteristically also in the tubulo-interstitial compartment (57.4\%) and extraglomerular vessels (64.4\%).

Other pathogenetic immune mechanisms, e.g. anti-phospholipid antibodies and anti-neutrophil cytoplasmatic antibodies, appear to be additionally involved, especially in triggering thrombosis and/or necrotizing glomerular lesions, respectively. Furthermore, nonimmune mechanisms, hyperperfusion and hyperfiltration associated with arterial hypertension and severe proteinuria as well as hyperlipidemia, play a more or less significant role in the progression of lupus renal disease. Several 
parameters provided by semiquantitative evaluation of the kidney biopsy by different techniques have been established to be not only of prognostic significance but also of particular clinical importance in decision making about the most appropriate therapeutic approach in SLE patients.

The now widely accepted original WHO classification of lupus glomerulonephritis (GN) basically separates normal glomerular histology class I (14-5.2\%), mild mesangiopathy class II (40-15.0\%) and mostly fairly indolent membranous GN class V (31-11.6\%) from severe forms of renal disease, strictly requiring immunosuppressive therapy, focal segmental class III (65-24.3\%) and particularly diffuse proliferative class IV (117-43.8\%) lupus GN. Up to $50 \%$ of WHO class transformations have been demonstrated by our biopsy follow-up study of 155 SLE patients treated according to agreed standards. By considering additionally the histomorphologic pattern of glomerular inflammatory reaction in our 370 biopsy cases, mixed membranous and proliferative type of GN was confirmed to be particularly characteristic for SLE (141-38.1\%). Furthermore, our studies provided evidence for a heterogeneity of GN types related at least to some extent to differences in pathogenesis in WHO classes III and IV.

A mesangial-transmembranous immune deposit distribution pattern has been found characteristic for SLE (55.9\%) especially when the deposits contain "fingerprints" $(17.3 \%)$. Our studies confirmed that prominent subendothelial immune deposits are among the most objective evidence of the activity of lupus GN. Furthermore, we share the opinion that kidney biopsy monitoring of the activity index and chronicity index, introduced in the early eighties into clinical trials by a group from NIH, can usefully contribute to planning the most appropriate therapy and biopsy follow-up evaluation.

Various immune mechanisms, including those related to extraglomerular deposits, as well as nonimmune mechanisms, play a role in the development and progression of tubulo-interstitial lesions, which have been confirmed to correlate best with the clinical parameters of renal insufficiency.

Extraglomerular vascular changes of various histopathological characteristics and pathogenesis have been far less extensively studied than glomerular despite the fact that they may also significantly influence the course and prognosis of lupus GN. Three main forms of vascular lesions were confirmed in our study of kidney tissue samples in 261 SLE patients 1. immune deposit (153-56.4\%) accompanied by lupus microangiopathy, uncomplicated and hyalinizing, and vasculitis 2. premature arteriosclerosis (101-38.0\%) and 3. thrombosis and thrombotic microangiopathy frequently related to positive anti-cardiolipin antibodies in the sera $(11-4.1 \%)$.

\section{REFERENCES}

1 Ferluga $D$, Jerše $M$, Vizjak $A$, et al. Correlation among WHO classes, histomorphologic patterns of glomerulonephritis and glomerular immune deposits in SLE. Wien Klin Wochenschr. 2000;112:692-701

2 Kashgarian M. The role of the kidney biopsy in the treatment of lupus nephritis. Ren Fail. 1996;18:765-73

\section{SP0101 CURRENT STANDARD TREATMENT FOR LUPUS NEPHRITIS}

DT Boumpas. Divisions of Internal Medicine and Rheumatology, Clinical Immunology and Allergy University of Crete, Medical School, Heraklion, Greece

10.1136/annrheumdis-2001.45
Glomerulonephritis is a major cause for morbidity in systemic lupus erythematosus (SLE). In this disease, immune complex formation/deposition in the kidney results in intraglomerular inflammation with recruitment of leukocytes, and activation and proliferation of resident renal cells. Intense injury may destroy resident renal cells by necrosis or apoptosis resulting in fibrinoid necrosis. When injury is less intense, endocapillary cells respond by proliferating and production of extracellular matrix (proliferative lesions).

Determination of disease severity: Renal biopsy, examination of the urine sediment and measurement of C3 levels (and to a lesser degree of anti-DNA titers) are essential for the management of lupus nephritis. Treatment depends on the severity of the disease. Accordingly disease severity is determined by the presence or absence of high-risk factors. These include demographic (male gender, black race), clinical (failure to acheive response or marked delay in response, multiple relapses, pregnancy), laboratory (impaired renal function, severe anaemia with hematocrit less than 26\%) and histologic features (mixed membranous and proliferative or proliferative nephritis; cellular crescents and/or fibrinoid necrosis; and moderate to high degrees of interstitial fibrosis and/or tubular atrophy).

Treatment: Patients with mild proliferative disease without risk factors are usually treated with corticosteroids alone or in combination with azathioprine. If the disease does not remit within 3-4 months, cytotoxic drugs such as cyclophoshamide or newer agents such as mycophenolate may be tried. For patients with moderate to severe proliferative nephritis, controlled trials have shown that pulse cyclophosphamide is the treatment of choice. Long-term follow-up of patients participating in these controlled trials suggest that combining pulse cyclophoshamide with pulse methylprednisolone increases efficacy but not toxicity. In general cytotoxic therapy continues 1 year beyond remission as shorter courses of cyclophosphamide have an increased risk for flares. Induction of response with cyclophosphamide followed by maintenance with agents such as azathioprine or cyclosporine is under investigation. For lupus membranous nephritis steroids, pulse cyclophospamide therapy or cyclosporine may be used. Relapse rates are high when cyclosporine is discontinued. In addition to immunosuppressive therapy, aggressive management of co-morbid conditions (hypertension, dyslipidemia, osteoporosis) is of paramount importance.

Response rates and flares: Rates of clinical response and flares vary in different studies according to type of nephritis, treatment regimen, duration of therapy, and definition of clinical response and flare used. Flares pose a significant problem because of the risk for renal function deterioration due to cumulative damage as well as cumulative toxicities due to additional immunosuppressive therapy. Patients with nephritic flares (defined as increase in plasma creatinine level and reappearance of active nephritic urinary sediment), are more likely to progress to endstage renal disease in spite of additional immunosuppressive therapy.

Side effects: Pulse cyclophosphamide is associated with an increased risk for herpes zoster infections on the short-term and with sustained amenorrhea or azoospermia on the long-term. Gonadotropin-releasing hormone agonist $(\mathrm{GnRH}-\mathrm{a})$ may prevent accelerated recruitment and depletion of ovarian follicles (via suppression of the gonadotrophin production in the pituitary gland) and therefore protect against premature ovarian failure. In a small case series, testosterone was found to be effective in preserving fertility in patients with nephrotic syndrome treated with a short course of cyclophosphamide. 